\title{
science
}
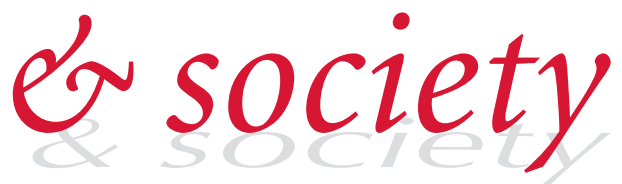

\section{Resistance redux}

\author{
Infectious diseases, antibiotic resistance and the future of mankind
}

\author{
Julian Davies
}

7 he human species is one of the youngest forms of life on earth, yet it has become the dominant force in evolution. Most of the main environmental problems-global warming, pollution, loss of biodiversity, land erosion and the emission of greenhouse gases-are caused, either directly or indirectly, by human activities. Optimists hope that these huge environmental changes might be at least partly reversible, if humans agree to take massive and concerted corrective action sooner rather than later. Pessimists - or perhaps realists - hold that it might already be too late, and that we are markedly changing the physical and biological environment with as yet unclear repercussions for humanity.

In any case, even if humans are the dominant species on our planet, they are not the most important from a purely biological point of view. The omnipresent microbial population is not only the oldest form of life on Earth, having emerged more than 3 billion years ago, but also the most numerous and biologically diverse, and the most able to adapt to environmental changes. According to many pundits, microbes will be the sole survivors of any global catastrophe short of the complete destruction of all life on Earth.

$\mathrm{H}$ umans are essentially interlopers in a microbial world. Our evolution has been completely dependent on microbes; this holds true for all of the commensal bacteria that make up the human microbiome, as well as the microorganisms in the external environment. In fact, all living organisms rely on the presence and activity of microbes for their survival, just as viruses and bacteria rely on favourable hosts and environments for growth and reproduction. From the point of view of a microbe, the human body is just one such host that can supply its guests with the proverbial 'free lunch'.

\section{According to many pundits, microbes will be the sole survivors of any global catastrophe short of the complete destruction of all life on Earth}

During the course of human-microbe acclimatization, humans have become dependent on microbes for various vitamins and other essential co-factors, among other things. In return, our bodies furnish these commensal populations with a rich source of nutrition, a warm place to live and, possibly, protection from predators. The sequencing of the human genome is still not complete and the most difficult partsequencing and deciphering the nature and roles of the microbiome-has only just begun; the bacterial genes residing in the human body outnumber the human genes by an estimated 100-to-1.

Of course, this human-microbial cooperation is not always beneficial to us. Indeed, a small number of the thousands of different types of bacteria that colonize the human body can cause disease with often detrimental effects for the host. The resulting bacterial and fungal infections are genetically and biochemically complex, and most involve interactions with a host that has been weakened or damaged by injury, age or trauma. Much of the history of medicine has therefore been a search for ways to battle the pathogenic organisms - syphilis, leprosy, typhus, consumption, and other assorted poxes and plagues - which have caused more death and suffering throughout human history than wars, famines and environmental disasters combined.

Given the ubiquity of the microbial world, it is not surprising that the main weapon in the treatment of infectious diseases came from the discovery that a range of microbial chemicals - antibiotics - can kill other microbes or inhibit their growth. The scientific basis for such apparent -cidal tendencies is still not understood; some of these naturally occurring small molecules might have evolved for protective purposes, but most are likely to have evolved for modulating inter-microbial communication. This chemical cross-talk depends on the concentrations of these small molecules; a given molecule acts as a messenger at low concentrations and exerts antibiotic activity at higher concentrations. Whatever their natural function, the discovery of microbial products with inhibitory or -cidal activity against laboratory strains of bacterial pathogens initiated the era of antibiotics approximately 60 years ago.

I t should be noted, however, that the incidence of infectious disease was already on the decline in much of the industrial world by the 1940s, owing to better sanitation, improved disinfection in hospitals, and overall improvements in personal hygiene and living standards. The groundbreaking work by Louis Pasteur (1822-1895), Robert Koch (1843-1910) and their disciples led to the isolation of the major pathogens and proved the role of 'germs' in disease. This increased understanding of microbiology translated into better health care, and successful publichealth efforts to reduce disease transmission and burden.

The discovery of penicillin by Alexander Fleming (1881-1955), followed by streptomycin, tetracycline and other antimicrobials, completely revolutionized medicine 
by providing physicians with effective therapeutic options against a range of bacterial diseases, many of which-such as the widely feared 'White Plague' (tuberculosis)—had been scourges for millennia. Antibiotics also became effective treatments for common sexually transmitted diseases such as syphilis and gonorrhoea (Fig 1). The introduction of antibiotics allowed physicians to markedly reduce human morbidity and mortality from most of the common bacterial and fungal infections. There were, however, forebodings of future problems: from the time when antibiotics were first used, scientists observed the development of resistance; however, the subsequent universal dependence on the curative powers of these agents led the scientific and medical community to underestimate the potential scale of the problem. By the 1970s, it was widely believed that infectious diseases were finally under control (Davies, 2007).

During the 1950s, the chemical industry, which until then had been largely concentrated on the production of fertilizers, dyes and other commodities, underwent a major transformation towards a pharmaceutical industry that involved the large-scale fermentation of bacterial and fungal strains to produce antimicrobial agents. The antibiotic era was characterized by mass production and mass utilization. In the early 1940s, less than 100 tons of penicillin was made in the USA annually; today, the figure is several orders of magnitude more, and the same holds true for every other therapeutically useful antibiotic. With mass production, prices dropped accordingly. Penicillin, which was nearly priceless in 1940, cost US\$20 for a single dose by 1943, 55 cents in 1946 and today probably costs less than the glass vials in which it is shipped.

\section{$\mathrm{T}$} he increased production and decreasing prices of antibiotics encouraged additional applications outside the medical setting. Low concentrations of antibiotics added to animal feeds were found to promote the growth of cattle, pigs and chickens, thereby resulting in increased economic turnover and profits for the meat industry-although this practice also accelerates antibiotic resistance. At present, approximately $50 \%$ of all antibiotics produced worldwide are used for non-human purposes, notably in agriculture and aquaculture. It is not surprising that the development of resistance to antibiotics shows a direct correlation with

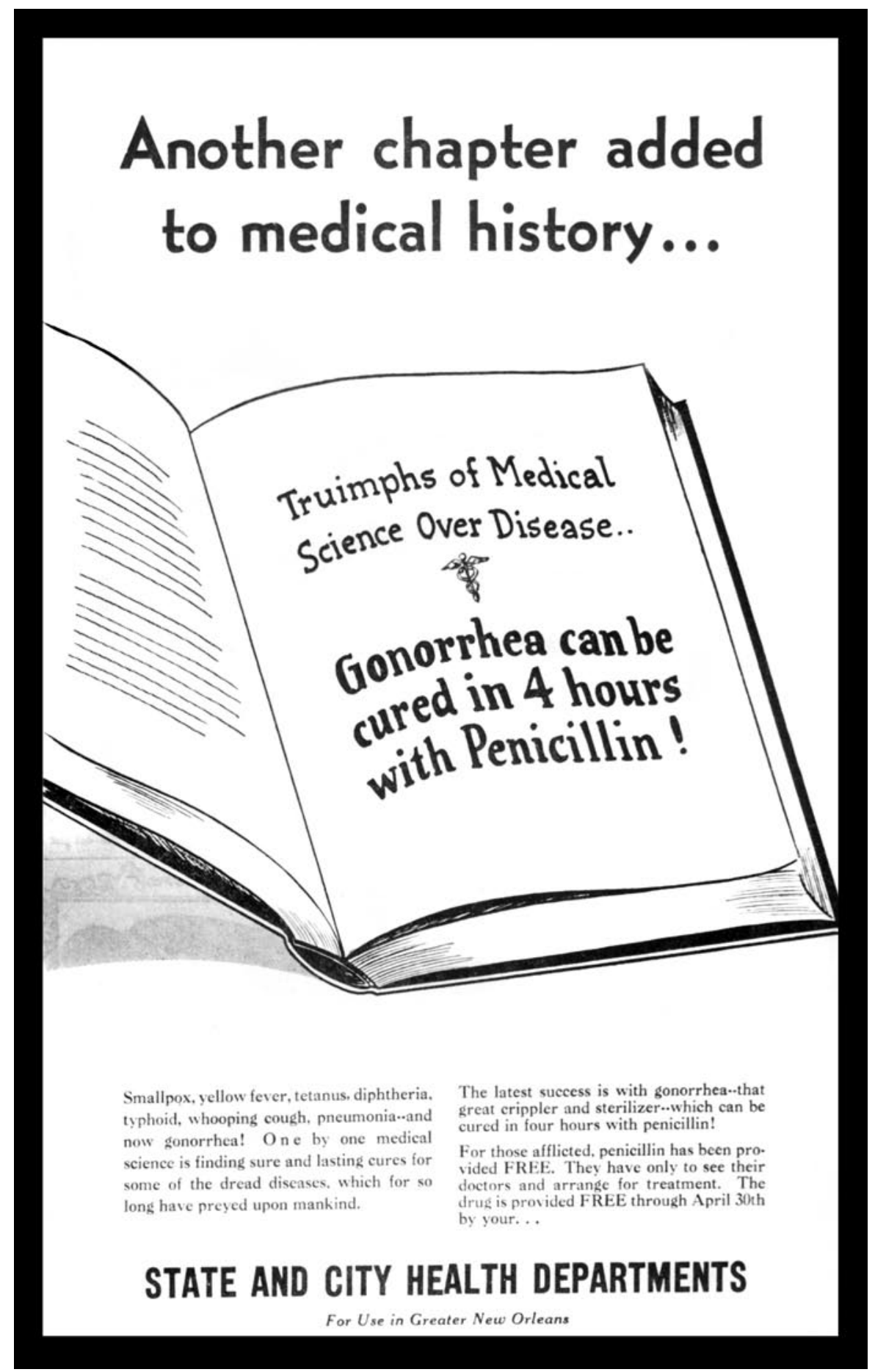

Fig 1 | A poster from the 1940s promoting penicillin as a treatment for gonorrhea. Today, strains of Neisseria gonorrhoeae are increasingly resitant to antibiotic treatment. Image credit: Centres for Disease Control and Prevention (CDC: Atlanta, GA, USA)

the extent of antibiotic use. Awareness of this problem has increased over the years and many countries have mounted efforts to educate the public about the threat posed by resistant bacteria, and have instituted measures to control antibiotic use. As long ago as 1969, the Swann report, which was published in the UK, recommended 
restricting the use of antibiotics in animal feeds (Swann, 1969). Recent progress is most evident in The Netherlands and the Scandinavian countries, where the use of antibiotics to promote growth in animals has been effectively curtailed. Reduced levels of antibiotic resistance in animals and farm workers indicate the success of these measures; however, despite such controls, resistance has not been eliminated and outbreaks of resistant pathogens still occur frequently. Cases of pig-related community-acquired multi-resistant Staphylococcus aureus (CAMRSA) in The Netherlands confirm the inevitability of antibiotic resistance-bacteria are omnipotent.

\section{...from the point of view of a microbe, the human body is just one such host that can supply its guests with the proverbial 'free lunch'}

The problem of antibiotic resistance is not only man-made; resistance genes were already present in the bacterial population long before humans started to use antibiotics to fight bacterial infections (Abraham \& Chain, 1940). The widespread resistance due to the cefotaxime (CTX-M) $\beta$-lactamases is a good example; the ancestral gene is thought to have been picked up from a strain of Kluyvera sp. and subsequently transmitted to other bacteria (Barlow et al, 2008). As a result of the extensive use of certain classes of antibiotics in the past 20 years, this gene has undergone many mutations and gene transfers, under increasing antibiotic selection, and has evolved into the family of extended spectrum $\beta$-lactamases (ESBLs). There is now a veritable pandemic with hundreds of different CTX-Ms being found in bacterial pathogens around the world. This is natural protein engineering on a grand scale, which David Livermore refers to as "the zeitgeist of resistance" (Livermore, 2007). Although these genes were first detected in bacterial strains in hospital settings, resistant bacteria with various CTX-M genes are being found increasingly in the community. Was this series of events inevitable? Could it have been stopped? After all, $\beta$-lactamases had been recognized for many years. The costs in terms of mortality, morbidity and economic losses are significant, but hard to estimate. So, despite an armamentarium of antibacterial, antifungal and antiviral agents for the treatment of most infectious diseases, the biggest threats today are resistant pathogens, especially those in hospitals.

T he cloud of antibiotic resistance does have a surprising 'silver lining' as it has also had enormous benefits for modern biology and, indeed, the human condition-although not without some controversy. The identification of transmissible antibiotic resistance in the mid1950s subsequently shed light on the evolutionary processes in cell development. Studies of plasmids, transposable elements, integron systems and illegitimate-recombination mechanisms have demonstrated the important roles of gene trafficking over billions of years. In particular, recent research has revealed the roles of these mobile elements in bacterial genome evolution in response to the selective pressures of antibiotics, and of toxic compounds such as heavy metals and other xenobiotics.

There have also been important practical applications. Recombinant DNA technology grew out of the use of antibiotic-resistance plasmids as vectors. For example, in their Nobel Prize-winning research, Mario Cappechi and Oliver Smithies used an antibiotic-resistance gene encoding kanamycin phosphotransferase, coupled with an appropriate broadspectrum antibiotic (for example, geneticin) to generate large libraries of gene knockouts in mice. Similar techniques have advanced many other aspects of both basic research and industrial processes, and now offer considerable promise for stem-cell-based technology applied to human disease. Genetic tools are now available for the introduction of many different types of genes into microbes, plants, animals and humans; recombinant bacteria containing resistance markers are used to produce large amounts of pure therapeutic proteins and vaccines. Without the discovery of transferable antibiotic resistance in bacteria, which allowed the design and use of gene vectors, genetic engineering would probably not have advanced to such a sophisticated level.

\section{Without the discovery of} transferable antibiotic resistance in bacteria $[. .$.$] genetic$ engineering would probably not have advanced to such a sophisticated level
N otwithstanding these enormous advances in molecular biology and the concomitant practical applications, the rise of antibiotic resistance has serious economic and social consequences. Resistant bacterial and fungal infections are most frequently hospital-acquired (nosocomial). This is cause for great concern: patients go to hospitals to be cured, not to become ill and die. In the USA alone, almost 100,000 people die of hospital-acquired infections every year, and the annual financial burden on the health-care system increased by an additional US $\$ 10$ billion owing to MRSA infections in 2005 (Klein et al, 2007). Hospitals and other places of care have become breeding grounds for multidrug-resistant infectious bacteria.

\section{Antibiotic resistance has essentially become a crime, at least in the USA}

Numerous patients, prisoners and cruiseship passengers who contracted MRSA or other infections while being 'confined' have successfully pursued lawsuits claiming criminal negligence by the respective hospital, prison or travel organizers. Lawyers are now advertising their services on the Internet to victims of nosocomial microbial infection. More importantlyand perhaps more consequentially - the US Government has also responded in respect to Medicare, the national health insurance scheme. Starting in 2009, claims by patients to cover the increased cost of treatment for hospital-acquired diseases such as bacterial infection will no longer be accepted. Nosocomial multidrug-resistant infections that require extended hospital care will be considered as medical malpractice, in the same category as the non-removal of surgical instruments. The state of New Jersey has already enacted such a bill and others are considering similar action. These weighty developments are likely to trigger protective action from hospitals and the medical profession. The costs of quarantine will add to the costs of treatment. Antibiotic resistance has essentially become a crime, at least in the USA.

For many years, antibiotic-resistant pathogens have been recognized as one of the main threats to human survival, as some experts predict a return to the pre-antibiotic era. So far, national efforts 


\section{...microbes are formidable adversaries and, despite our best efforts, continue to exact a toll on the human race}

to exert strict control over the use of antibiotics have had limited success and it is not yet possible to achieve worldwide concerted action to reduce the growing threat of multi-resistant pathogens: there are too many parties involved. Furthermore, the problem has not yet really arrived on the radar screen of many physicians and clinicians, as antimicrobials still work most of the time-apart from the occasional news headline that yet another nasty superbug has emerged in the local hospital. Legislating the use of antibiotics for nontherapeutic applications and curtailing general public access to them is conceivable, but legislating the medical profession is an entirely different matter.

In order to meet the growing problem of antibiotic resistance among pathogens, the discovery and development of new antibiotics and alternative treatments for infectious diseases, together with tools for rapid diagnosis that will ensure effective and appropriate use of existing antibiotics, are imperative. How the health services, pharmaceutical industry and academia respond in the coming years will determine the future of treating infectious diseases. This challenge is not to be underestimated: microbes are formidable adversaries and, despite our best efforts, continue to exact a toll on the human race.

\section{ACKNOWLEDGEMENTS}

I appreciate the help of D. Davies in the preparation of this manuscript. Work on antibiotic resistance in my laboratory is supported by the Canadian Institutes for Health Research.

\section{REFERENCES}

Abraham EP, Chain E (1940) An enzyme from bacteria able to destroy penicillin. Nature 146: 837

Barlow M, Reik RA, Jacobs SD, Medina M, Meyer MP, McGowan JE Jr, Tenover FC (2008) High rate of mobilization for bla $\mathrm{CTX}_{\mathrm{M}} \mathrm{s}$. Emerg Infec Dis 14: 423-428
Davies J (2007) Microbes have the last word. EMBO Rep 8: 616-621

Klein E, Smith DL, Laxminarayan R (2007) Hospitalizations and deaths caused by methicillin-resistant Staphyloccus aureus, United States, 1999-2005. Emerg Infec Dis 13: 1840-1846

Livermore D (2007) The zeitgeist of resistance. J Antimicrob Chemother 60: i59-i61

Swann MM (1969) Report of the Joint Committee on the Use of Antibiotics in Animal Husbandry and Veterinary Medicine. London, UK: Her Majesty's Stationary Office

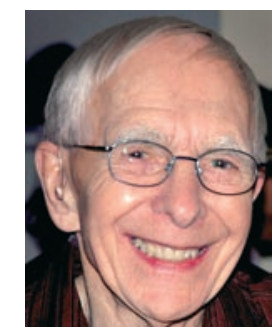

Julian Davies is in the Department of Microbiology and Immunology at the University of British Columbia in Vancouver, Canada. E-mail: jed@interchange.ubc.ca

doi:10.1038/embor.2008.69 